\title{
XMM-Newton slew survey discovery of the nova XMMSL1 J070542.7-381442 (V598 Puppis)
}

\author{
A. M. Read ${ }^{1}$, R. D. Saxton ${ }^{2}$, M. A. P. Torres ${ }^{3}$, P. Esquej ${ }^{4}$, E. Kuulkers ${ }^{2}$, \\ P. G. Jonker ${ }^{5,3}$, J. P. Osborne ${ }^{1}$, M. J. Freyberg ${ }^{4}$, and P. Challis ${ }^{3}$ \\ 1 Dept. of Physics and Astronomy, Leicester University, Leicester LE1 7RH, UK \\ e-mail: amr30@star.le.ac.uk \\ 2 ESA/ESAC, Apartado 78, 28691 Villanueva de la Cañada, Madrid, Spain \\ Harvard-Smithsonian Center for Astrophysics, Cambridge, MA 02138, USA \\ 4 Max-Planck-Institut für extraterrestrische Physik, 85748 Garching, Germany \\ 5 SRON, Netherlands Institute for Space Research, 3584 CA, Utrecht, The Netherlands
}

Received 25 January 2008 / Accepted 2 March 2008

ABSTRACT

\begin{abstract}
Aims. In an attempt to catch new X-ray transients while they are still bright, the data taken by XMM-Newton as it slews between targets are being processed and cross-correlated with other X-ray observations as soon as the slew data appear in the XMM-Newton archive.

Methods. A bright source, XMMSL1 J070542.7-381442, was detected on 9 Oct. 2007 at a position where no previous X-ray source had been seen. The XMM slew data and optical data acquired with the Magellan Clay $6.5 \mathrm{~m}$ telescope were used to classify the new object.

Results. No XMM slew X-ray counts are detected above $1 \mathrm{keV}$ and the source is seen to be $\sim 750$ times brighter than the ROSAT All-Sky Survey upper limit at that position. The normally $m_{\mathrm{V}} \sim 16$ star, USNO-A2.0 0450-03360039, which lies 3.5" from the X-ray position, was seen in our Magellan data to be very much enhanced in brightness. Our optical spectrum showed emission lines that identified the source as a nova in the auroral phase; hence, this optical source is undoubtedly the progenitor of the X-ray source - a new nova (now also known as V598 Pup). The X-ray spectrum indicates that the nova was in a super-soft state (with $k T_{\text {eff }} \approx 35 \mathrm{eV}$ ). We estimate the distance to the nova to be $\sim 3 \mathrm{kpc}$. Analysis of archival robotic optical survey data shows a rapid-decline light curve consistent with what is expected for a very fast nova.

Conclusions. The XMM-Newton slew data present a powerful opportunity to find new X-ray transient objects while they are still bright. Here we present the first such source discovered by the analysis of near real-time slew data.
\end{abstract}

Key words. stars: novae, cataclysmic variables - stars: individual: V598 Puppis - surveys - X-rays: general

\section{Introduction}

To date, the publicly available XMM-Newton slew data cover over $25 \%$ of the sky, while the soft band $(0.2-2 \mathrm{keV})$ slew sensitivity limit $\left(6 \times 10^{-13} \mathrm{erg} \mathrm{cm}^{-2} \mathrm{~s}^{-1}\right)$ is close to that of the RASS. (The hard-band [2-12 keV] limit is $4 \times 10^{-12} \mathrm{erg} \mathrm{cm}^{-2} \mathrm{~s}^{-1}$.) For details of the slew data and catalogue and the first science results, see Saxton et al. (2008) and Read et al. (2006). The near real-time comparison of XMM-Newton slew data with ROSAT data is now giving, for the first time, the opportunity of finding all manner of high-variability X-ray objects, e.g. tidal disruption candidates (Esquej et al. 2007), AGN, blazars, and also Galactic sources such as novae, flare stars, cataclysmic variables, and eclipsing X-ray binaries. It is only with a large-area survey, such as the XMM-Newton Slew Survey, that such rare events have a chance of being caught - within the first slew catalogue (XMMSL1, covering 14\% of the sky; Saxton et al. 2008), $\sim 40$ individual slew sources are seen at fluxes $>20$ times greater than their corresponding ROSAT counterparts or $2 \sigma$ upper limits (assuming a $70 \mathrm{eV}$ black body model with Galactic $N_{\mathrm{H}}$ ). $55 \%$ of these are believed to be new X-ray transients.

In an effort to find transient X-ray sources while they remain active, we are now attempting to perform the slew data acquisition, analysis, and source-searching as quickly as possible. Catalogue cross-correlations with RASS and ROSAT pointed data fluxes and upper limits are swiftly performed to identify highly variable X-ray candidates. Slew datasets appear in the XMM science archive (XSA) typically $~ 10$ days after the slew has been performed. This systematic processing of the most recent data has been going on since October 2006.

One such rare event, XMMSL1 J070542.7-381442, was discovered in an XMM slew from 2007 Oct. 09. Here we describe the XMM-Newton slew observations, the identification of the optical counterpart, and a spectral confirmation of the source as a nova in the auroral phase. The optical lightcurve of the source up to six months after outburst is also presented. Since the discovery, follow-up observations have been made with XMM-Newton and with Swift (Read et al. 2007b), and the X-ray flux is observed to have declined continually. The discussion of these observations is deferred to a later paper.

\section{XMM-Newton slew observations}

XMMSL1 J070542.7-381442 was discovered at an X-ray position of 07:05:42.7-38:14:42 (J2000; error radius: $8^{\prime \prime}$ ) in slew 9143400002 from XMM revolution 1434, made in the EPIC-pn 


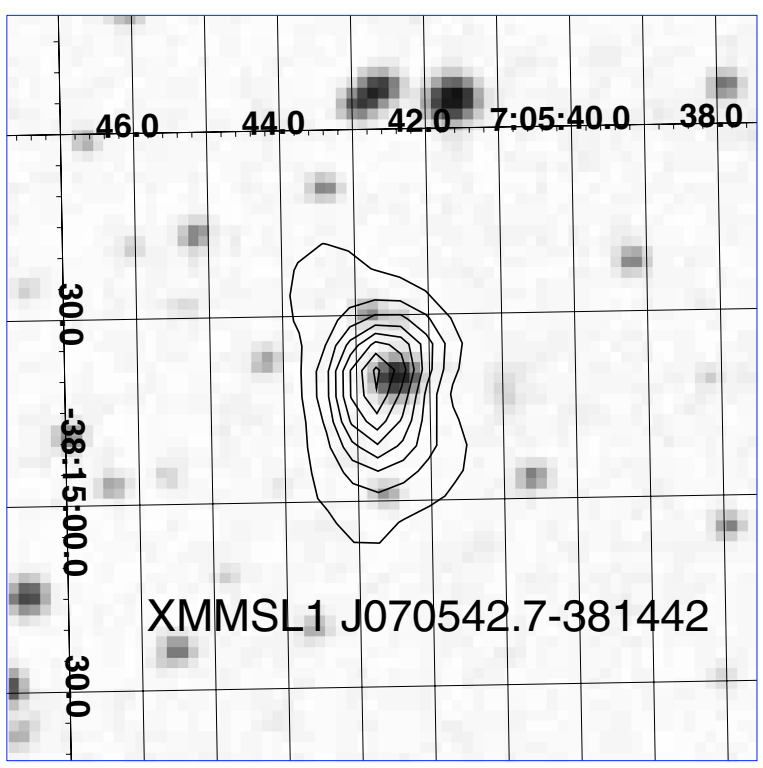

Fig. 1. Contours $\left(16.8,33.6,50.4\right.$ etc. cts $\left.\operatorname{arcsec}^{-2}\right)$ of $\left(4^{\prime \prime} F W H M\right)$ Gaussian-smoothed XMM-Newton slew data (EPIC-pn; 0.2-2 keV) from XMMSL1 J070542.7-381442 superimposed on an optical DSS image. A slight elongation of the X-ray emission along the slew direction (due partly to the source slew drift during an individual EPIC-pn cycle time $(73 \mathrm{~ms})$ and also to the PSF shape at this offaxis position) is evident. The progenitor, the $m_{\mathrm{V}} \sim 16$ point-like source, USNO-A2.0 0450-03360039, is clearly visible.

full-frame mode using the medium filter (Read et al. 2007a). Contours of the XMM-Newton slew data are shown superimposed on an optical DSS image in Fig. 1. This sky position has not yet been observed during any other XMM-Newton slew.

The source passed through the centres of CCDs 3 and 12 of the EPIC-pn detector in $14 \mathrm{~s}$, at a large off-axis angle (minimum $\approx 14^{\prime}$ ), such that an effective vignetting-corrected soft band $(0.2-2 \mathrm{keV})$ exposure time of $3.9 \mathrm{~s}$ was achieved. A total of 210 source counts lie within a radius of $20^{\prime \prime}$, yielding a (EPIC-pn: $0.2-2 \mathrm{keV}$ ) count rate of $54.5 \mathrm{ct} \mathrm{s}^{-1}$, after correcting for the encircled energy function. The high count rate indicates that the spectrum is affected by pile-up, though the effect here, far off-axis, is less than on-axis (the on-axis limit is $6 \mathrm{ct} \mathrm{s}^{-1}$ for EPIC-pn full-frame mode $\left.{ }^{1}\right)$. Some X-ray loading (Smith 2004), where events below the cut-off threshold sum together to produce artificial accepted events, is also present. These effects work to distort the spectrum and make quantitative spectral analysis difficult. One can minimize these effects by ignoring the central part of the PSF, and we therefore extracted a spectrum of the source from within an annulus of $5^{\prime \prime}-60^{\prime \prime}$ radius, centred on the source position. Just single events were selected, and these were spectrally grouped to give a minimum of 20 counts per bin. Slew data also still has unresolved problems associated with the motion of the source across the detector, so that approximations currently have to be made when calculating the associated effective area and detector response matrix files. To perform a qualitative spectral analysis, an effective area file, accounting for the removal of the piled-up core, was generated by averaging the individual core-removed effective area files at 9 different positions along the detector track made by the source. This takes the variations in the vignetting and the PSF into account to a good approximation. Individual BACKSCAL

\footnotetext{
1 http://xmm.esac.esa.int/external/xmm_user_support/ documentation/uhb_2.5/index.html
}

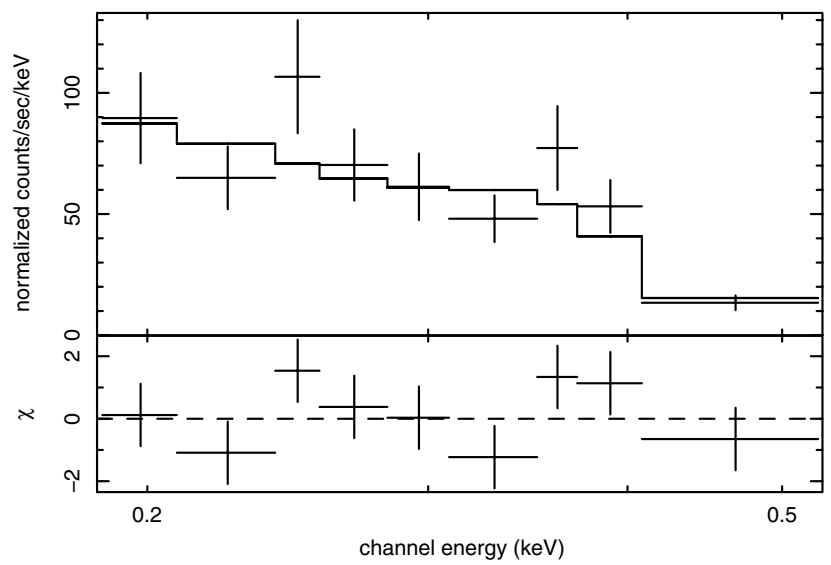

Fig. 2. The XMM-Newton slew spectrum of XMMSL1 J070542.7381442 , fit with a white dwarf atmosphere model of effective temperature $35 \mathrm{eV}$. The data and model are shown in the upper panel and the deviations of the data from the model are shown in the lower panel.

values have been set by hand, as have the EXPOSURE values, estimated by calculating the distance travelled by the source in detector coordinates and finding the time taken to do this given a $90 \mathrm{deg} \mathrm{h}^{-1}$ slew speed, then subtracting the appropriate fractions for chip gaps and bad pixels (calculating the exposure time from the source lightcurve, gives the same value to within a few tenths of a second). For the response matrix, we used the equivalent canned detector response matrix for the vignetting-weighted average source position, for single events and for Full Frame mode: epn_ff20_sY6_v6.9.rmf. A background spectrum was extracted from a much larger region close to the source and at a similar off-axis angle.

Simple power-law, blackbody, thermal Bremmstrahlung, and optically thin hot plasma models are unable to fit the spectrum adequately (all have a $\chi^{2} \sim 12$ for 6 degrees of freedom). Given that the source is identified as a nova (Sect. 3), a more physically realistic white dwarf atmosphere model of the type used to model the nova V1974 Cyg (Balman et al. 1998) was used, yielding an acceptable fit (reduced $\chi^{2} \approx 1.4$ for 6 degrees of freedom), an effective temperature of $35_{-1}^{+2} \mathrm{eV}$, and an $N_{\mathrm{H}}$ of $4.8_{-2.7}^{+5.7} \times$ $10^{20} \mathrm{~cm}^{-2}$ (errors $90 \%$ for one interesting parameter). The spectrum and model fit are shown in Fig. 2. The model normalization, corrected for the removal of the saturated PSF core, can be used to derive a distance to the source (assuming the emission to be sub-Eddington, and a typical emitting region of spherical radius $10^{9} \mathrm{~cm}$ ) of $\approx 4.3 \pm 2.3 \mathrm{kpc}$. A PIMMS ${ }^{2} \mathrm{v} 3.9 \mathrm{~d}$ conversion of the RASS $0.0076 \mathrm{ct} \mathrm{s}^{-1} 2 \sigma$ upper limit corresponds to an EPIC-pn limit $\sim 750$ times less than what is observed of $0.073 \mathrm{ct} \mathrm{s}^{-1}$, assuming the same spectral model.

\section{Optical observations}

The field containing XMMSL1 J070542.7-381442 was observed on 16 Nov. 2007 with the Magellan Clay $6.5 \mathrm{~m}$ telescope at Las Campanas Observatory. A bright, saturated optical counterpart was found with a position consistent with the $m_{\mathrm{V}} \sim$ 16 point-like source USNO-A2.0 0450-03360039 (07:05:42.5 $-38: 14: 39$; J2000), 3.5" from the slew position. On the basis of the positional coincidences, the USNO object was proposed as a possible progenitor of the nova (Read et al. 2007a; Torres et al. 2007). This claim was confirmed by the detection of the

\footnotetext{
${ }^{2}$ http://heasarc.nasa.gov/Tools/w3pimms.html
} 
radio counterpart to the slew source, which has a high-precision position consistent with the USNO object (Rupen et al. 2007).

Spectroscopic observations of the optical counterpart were obtained with the Low Dispersion Survey Spectrograph (LDSS-3), equipped with the 400 line $\mathrm{mm}^{-1}$ VPH ALL grism and a mask with a $1^{\prime \prime}$ width long-slit cut near the centre of the field of view. The detector was the STA0500A 4k $\times 4 \mathrm{k}$ unbinned CCD. This setup allowed us to cover the spectral range 3510-10620 $\AA$ with a dispersion of $2.0 \AA$ pix $^{-1}$ and a resolution of $\approx 8 \AA F W H M$. Several exposures $(2 \times 1 \mathrm{~s}, 1 \times 2 \mathrm{~s}$, $1 \times 5 \mathrm{~s}$, and $1 \times 60 \mathrm{~s}$ ) were obtained. These spectra were affected by 2 nd-order light contamination beyond $\sim 7000 \AA$. To obtain useful coverage in the red, we also acquired $1 \mathrm{~s}, 5 \mathrm{~s}$, and 60 s spectra with the OG590 order-blocking filter, giving useful 5800-10620 A wavelength coverage. The frames were reduced using standard routines in IRAF. The spectra were then extracted and wavelength-calibrated with the help of HeNeAr calibration lamp spectra. The instrumental response was corrected using spectroscopic standard stars observed with and without the blocking filter. The shape of the continuum is reliable except at the blue and red ends.

Blue and red spectra, obtained 163 days after the maximum optical brightness reported by Pojmanski et al. (2007) (see Sect. 4), are shown in Fig. 3. The data show emission lines characteristic of a late post-outburst nova spectrum. The strongest lines in the blue spectrum are [O III] $\lambda 5007,4959$ followed by $\mathrm{H} \alpha$ blended with [N II] $\lambda 6583$, [O III] $\lambda 4363$ (blended with $\mathrm{H} \gamma$ ), [N II] $\lambda 5755$, and $\mathrm{H} \beta$. The strongest emission feature in the red spectrum is due to [O II] $\lambda \lambda 7319-7330$ auroral transitions. The emission lines have an average $F W H M$ of $2070 \pm 50 \mathrm{~km} \mathrm{~s}^{-1}$, as derived from isolated line profiles and correcting for the instrumental broadening. The $\mathrm{H} \beta$ line is stronger than He II $\lambda 4686$. This characteristic, together with the presence of strong forbidden $[\mathrm{O}$ III $],[\mathrm{N} \mathrm{II}]$ and [O II] auroral lines, and weak Ne lines, suggests that this source was an Fe II nova (see e.g. Williams 1992). However, the data were acquired too long after maximum light to make an accurate classification possible. The nova was observed in the $\mathrm{A}_{0}$ auroral phase according to the Tololo classification (Williams et al. 1991, 1994) - any forbidden auroral transition at wavelengths $\lambda \lambda 3600-7600$ has a larger flux than the strongest non-Balmer permitted lines, and [O III] $\lambda 4363$ is the strongest auroral transition. The coronal [Fe X] $\lambda 6375$ line (if present) is weaker than [Fe VII] 26375 , excluding the possibility of a coronal stage.

\section{Optical light curve}

Analysis of archival robotic optical survey data from 3-min CCD exposures (pixel size 14."8), obtained with a $70 \mathrm{~mm}$ (200 mm focal length) $f / 2.8$ telephoto lens in the course of the All Sky Automated Survey (Pojmanski 2002) show that the visual magnitude of this source rose from $m_{\mathrm{V}} \gtrsim 14$ to $m_{\mathrm{V}} \approx 4.1$ between 2 Jun. (23:27 UT) and 5 Jun. (23:13 UT) 2007 and has declined since (see Fig. 4). The source was seen to be saturated in the June 5 observation, and it is thought that the source may have been $0.1-0.5 \mathrm{mag}$ brighter. The decline from outburst $(\gtrsim 2.4 \mathrm{mag}$ in 12 days, then a further 2.8 mag in 62 days) indicates that this is a nova of the very fast speed class (Warner 1995). We estimate $t_{2}$, the time that the light curve takes to decline 2 mag below maximum brightness as $9 \pm 1$ days. The later decay rate (mid-August onwards) is $1.36 \pm 0.04 \mathrm{mag}$ per 100 days (including errors to obtain a reduced $\chi^{2}$ of $\approx 1$ for the fit).
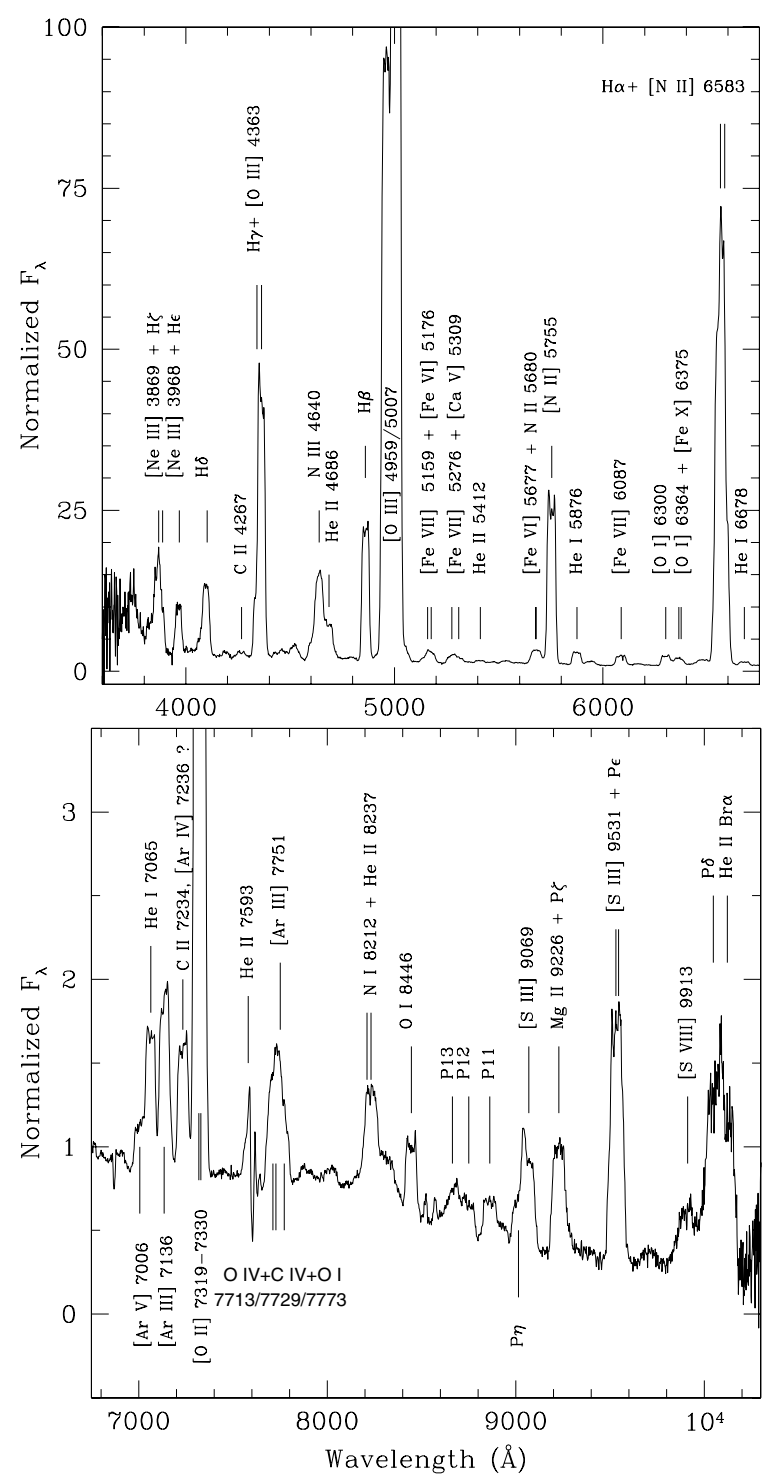

Fig. 3. Blue (top) and red (bottom) spectra of the optical counterpart to XMMSL1 J070542.7-381442 acquired on 16/11/07. The spectra have been normalized to unity at $\lambda 6750$. Stronger emission lines are identified. The absorption feature at He II $\lambda 7593$ is telluric in origin.

\section{Discussion}

As a confirmed classical nova, XMMSL1 J070542.7-381442 has been given the name V598 Puppis (Samus 2007). With a peak $m_{\mathrm{V}} \lesssim 4$, it is one of the brightest optical novae seen for many years. An $m_{\mathrm{V}} \lesssim 4$ nova has been discovered in eruption every $\approx 8$ years or so (Warner 1995), and the only novae discovered in recent decades of comparable peak optical magnitude are V382 Vel $(m \sim 3)$ and V1494 Aql $(m \sim 4)$, both discovered in 1999, and V1280 Sco $(m \sim 4)$, discovered in Feb. 2007.

Classical novae are usually discovered optically in the early phases of the outburst, since they are intrinsically optically bright and easily found in inexpensive wide-area shallow surveys. Unusually the optical outburst went unnoticed in this case, and V598 Pup was only discovered in X-rays during the later ( $\sim 100$ days after outburst), optically thin nebular phase, when classical novae are typically observed as soft X-ray sources.

On the basis of the optical spectrum, this nova was observed in the $\mathrm{A}_{0}$ auroral phase, and was likely a very fast, Fe II nova 


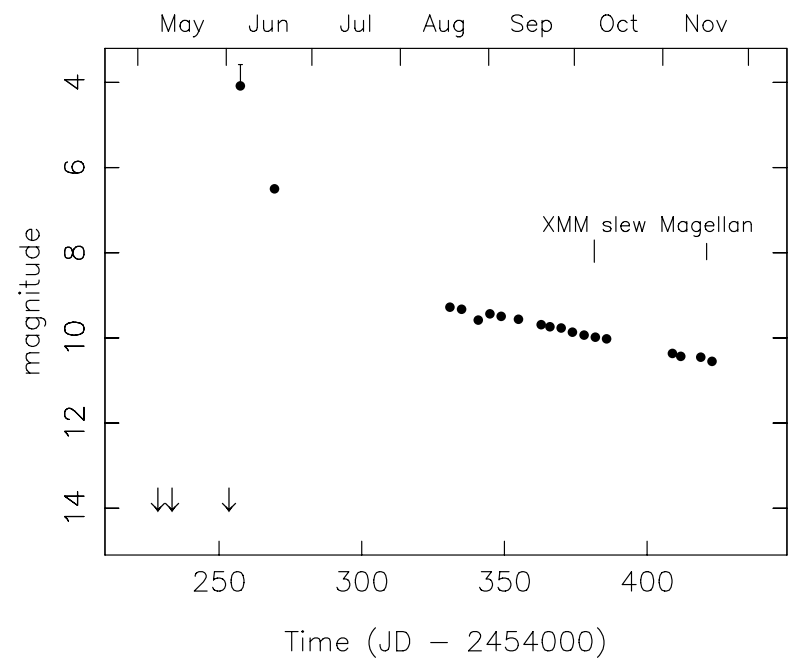

Fig. 4. $V$-band magnitudes from Pojmanski et al. (2007) of the optical counterpart to XMMSL1 J070542.7-381442. The 2007 dates of the XMM-Newton slew and Magellan observations are marked.

(Sect. 3 and Williams et al. 1991, 1994), though an accurate classification is no longer possible, this late after maximum brightness. The soft $\left(k T_{\text {eff }}=35 \mathrm{eV}\right) \mathrm{X}$-ray spectrum indicates that the nova was in a super-soft state (Pietsch et al. 2007). Such a state stems from nuclear burning on the white dwarf (Ness et al. 2007). Measurement of its intensity, duration, and temperature can constrain the distance to the nova and the mass of the white dwarf (e.g. Balman et al. 1998; Lanz et al. 2005). From the slew measurement of V598 Pup, we see that the delay from the outburst (2-5 Jun. 2007) to the onset of the X-ray supersoft state is $\$ 127$ days. This is short when compared with the $\sim 200$ days seen in V1974 Cyg (Krautter et al. 1996), 6 months of V382 Vel (Orio et al. 2002), and 6-8 months of V1494 Aql (Drake et al. 2003).

From the Galactic latitude and the fact that the Galactic scale height of white dwarfs is $<500$ pc (conservatively, Nelson et al. 2002), an upper limit to the distance of V598 Pup of $\approx 2.1 \mathrm{kpc}$ can be derived, consistent with the $4.3 \pm 2.3 \mathrm{kpc}$ estimated from the X-ray spectral fitting. Another way to estimate the distance is to use the relation between absolute magnitude at maximum brightness and $t_{2}$ (see e.g. Eq. (5.2) in Warner 1995). Using $t_{2}=$ $9 \pm 1$ days, we estimate $M_{\mathrm{V}}=-8.4 \pm 0.4$. With $A_{\mathrm{V}}=0.27_{-0.15}^{+0.31}$
(90\% error), as derived (Predehl \& Schmitt 1995) from $N_{\mathrm{H}}=$ $4.8_{-2.7}^{+5.7} \times 10^{20} \mathrm{~cm}^{-2}, M_{\mathrm{V}}=-8.4 \pm 0.4$, and peak $m_{\mathrm{V}}=4.1$, we derive a distance of $2.8_{-0.5}^{+0.8} \mathrm{kpc}$. An absolute magnitude of $M_{\mathrm{V}}=$ -8.4 would imply a peak luminosity $\sim 7$ times the Eddington luminosity for a $1 M_{\odot}$ white dwarf. This is quite typical of novae. The source had, at the time of the slew detection, an absorbed (0.2-2 keV) X-ray flux of $1.54_{-0.23}^{+0.08} \times 10^{-10} \mathrm{erg} \mathrm{cm}^{-2} \mathrm{~s}^{-1}$, corresponding to a $(0.2-2 \mathrm{keV}) \mathrm{X}$-ray luminosity of $1.6_{-0.2}^{+0.1} \times$ $10^{35} \mathrm{erg} \mathrm{s}^{-1}$, assuming a distance of $3 \mathrm{kpc}$, and a bolometric luminosity of $2.4_{-1.7}^{+6.4} \times 10^{36} \mathrm{erg} \mathrm{s}^{-1}$ (errors calculated at the boundary of the $90 \%$ region for two interesting parameters; $N_{\mathrm{H}}$ and $\left.k T_{\text {eff }}\right)$. This is at the lower end of the luminosities discussed e.g. in Orio et al. (2002) and Ness et al. (2007).

Acknowledgements. This research made use of the VIZIER database, operated at the CDS, Strasbourg, France. The XMM-Newton project is an ESA Science Mission with instruments and contributions directly funded by ESA Member States and the USA (NASA). We thank K. L. Page \& M. Modjaz for useful discussions, and the referee for useful comments that have improved the paper. A.M.R. \& J.P.O. acknowledge the funding support of PPARC/STFC, PE of MPE, and PGJ of the Netherlands Organisation for Scientific Research.

\section{References}

Balman, S., Krautter, J., \& Oegelman, H. 1998, ApJ, 499, 395 Drake, J. J., Wagner, R. M., Starrfield, S., et al. 2003, ApJ, 584, 448 Esquej, P., Saxton, R. D., Freyberg, M. J., et al. 2007, A\&A, 462, 49 Krautter, J., Oegelman, H., Starrfield, S., et al. 1996, ApJ, 456, 788 Lanz, T., Telis, G. A., Audard, M., et al. 2005, ApJ, 619, 517L

Nelson, C. A., Cook, K. H., Axelrod, T. S., et al. 2002, ApJ, 573, 644 Ness, J.-U., Schwartz, G. J., Retter, A., et al. 2007, ApJ, 663, 505 Orio, M., Parmar, A. N., Greiner, J., et al. 2002, MNRAS, 333, L11 Pietsch, W., Haberl, F., Sala, G., et al. 2007, A\&A, 465, 375 Pojmanski, G. 2002, Acta Astron., 52, 397

Pojmanski, G., Szczygiel, D., \& Pilecki, B. 2007, IAU Circ., 8899 Predehl, P., \& Schmitt, J. H. M. M. 1995, A\&A, 293, 889 Read, A. M., Saxton, R. D., Esquej, P., et al. 2006, PASJ, 58, 47 Read, A. M., Saxton, R. D., \& Esquej, P. 2007a, ATEL, 1282 Read, A. M., Saxton, R. D., Osborne, J. P., et al. 2007b, ATEL, 1301 Rupen, M. P., Dhawan, V., \& Mioduszewski, A. J. 2007, ATEL, 1305 Samus, N. N. 2007, IAU Circ., 8898

Saxton, R. D., Read, A. M., Esquej, P., et al. 2008, A\&A, accepted Smith, M. J. S. 2004, XMM-Newton Calibration technical note: XMM-SOC-CAL-TN-0050

Torres, M. A. P., Jonker, P. G., Challis, P., et al. 2007, ATEL, 1285 Warner, B. 1995, Cataclysmic Variable Stars (Cambridge)

Williams, R. E. 1992, AJ, 104, 725

Williams, R. E., Hamuy, M., Phillips, M. M., et al. 1991, ApJ, 376, 721 Williams, R. E., Phillips, M. M., \& Hamuay, M. 1994, ApJS, 90, 297 